\title{
On the nonlinear Hadamard-type integro-differential equation
}

Chenkuan $\mathrm{Li}^{*}{ }^{*}$ (D)

\author{
Correspondence: lic@brandonu.ca \\ 1 Department of Mathematics and \\ Computer Science, Brandon \\ University, Brandon, Manitoba \\ R7A 6A9, Canada
}

\begin{abstract}
This paper studies uniqueness of solutions for a nonlinear Hadamard-type integro-differential equation in the Banach space of absolutely continuous functions based on Babenko's approach and Banach's contraction principle. We also include two illustrative examples to demonstrate the use of main theorems.

MSC: 34A08; 34A12

Keywords: Hadamard-type fractional derivative; Hadamard-type fractional integral; Babenko's approach; Multivariate Mittag-Leffler function; Absolute continuity; Banach's contraction principle
\end{abstract}

\section{Introduction}

The Hadamard fractional integration and differentiation are based on the $n$th integral of the form $[1,2]$

$$
\begin{aligned}
\left(\mathcal{J}_{a+, \mu}^{n} u\right)(x) & =x^{-\mu} \int_{a}^{x} \frac{d t_{1}}{t_{1}} \int_{a}^{t_{1}} \frac{d t_{2}}{t_{2}} \cdots \int_{a}^{t_{n-1}} t_{n}^{\mu} u\left(t_{n}\right) \frac{d t_{n}}{t_{n}} \\
& =\frac{1}{(n-1) !} \int_{a}^{x}\left(\frac{t}{x}\right)^{\mu}\left(\log \frac{x}{t}\right)^{n-1} u(t) \frac{d t}{t}
\end{aligned}
$$

and the corresponding derivative

$$
\begin{aligned}
& \left(\mathcal{D}_{a+, \mu}^{1} u\right)(x)=((\delta+\mu) u)(x)=x u^{\prime}(x)+\mu u(x), \quad \delta=x \frac{d}{d x}, \\
& \mathcal{D}_{a+, \mu}^{n} u=\mathcal{D}_{a+, \mu}^{1}\left(\mathcal{D}_{a+, \mu}^{n-1} u\right), \quad n=2,3, \ldots,
\end{aligned}
$$

where $\log (\cdot)=\log _{e}(\cdot), 0<a<x<b$, and $\mu \in R$.

The fractional version of the Hadamard-type integral and derivative are given by

$$
\left(\mathcal{J}_{a+, \mu}^{\alpha} u\right)(x)=\frac{1}{\Gamma(\alpha)} \int_{a}^{x}\left(\frac{t}{x}\right)^{\mu}\left(\log \frac{x}{t}\right)^{\alpha-1} u(t) \frac{d t}{t}, \quad \alpha>0
$$

(c) The Author(s) 2021. This article is licensed under a Creative Commons Attribution 4.0 International License, which permits use, sharing, adaptation, distribution and reproduction in any medium or format, as long as you give appropriate credit to the original author(s) and the source, provide a link to the Creative Commons licence, and indicate if changes were made. The images or other third party material in this article are included in the article's Creative Commons licence, unless indicated otherwise in a credit line to the material. If material is not included in the article's Creative Commons licence and your intended use is not permitted by statutory regulation or exceeds the permitted use, you will need to obtain permission directly from the copyright holder. To view a copy of this licence, visit http://creativecommons.org/licenses/by/4.0/ 
and

$$
\left(\mathcal{D}_{a+, \mu}^{\alpha} u\right)(x)=x^{-\mu} \delta^{n} x^{\mu}\left(\mathcal{J}_{a+, \mu}^{n-\alpha} u\right)(x)
$$

where $n=[\alpha]+1$, and $[\alpha]$ being integral part of $\alpha$.

When $0<\alpha<1$, the fractional derivative turns out to be

$$
\begin{aligned}
\left(\mathcal{D}_{a+, \mu}^{\alpha} u\right)(x) & =x^{-\mu} \delta x^{\mu}\left(\mathcal{J}_{a+, \mu}^{1-\alpha} u\right)(x) \\
& =\frac{1}{\Gamma(1-\alpha)} x^{-\mu+1} \frac{d}{d x} \int_{a}^{x} t^{\mu-1}\left(\log \frac{x}{t}\right)^{-\alpha} u(t) d t
\end{aligned}
$$

In particular, for $\alpha=1$,

$$
\left(\mathcal{J}_{a+, \mu} u\right)(x)=\left(\mathcal{J}_{a+, \mu}^{1} u\right)(x)=\frac{1}{\Gamma(\alpha) x^{\mu}} \int_{a}^{x} t^{\mu-1} u(t) d t
$$

which leads to defining the space $X_{\mu}[a, b]$ of those Lebesgue measurable functions $u$ on $[a, b]$ for which $x^{\mu-1} u(x)$ is absolutely integrable [2]:

$$
X_{\mu}[a, b]=\left\{u:[a, b] \rightarrow C \text { and }\|u\|_{X_{\mu}}=\int_{a}^{b} x^{\mu-1}|u(x)| d x<\infty\right\} .
$$

Let $\mathrm{AC}[a, b]$ be the set of absolutely continuous functions on $[a, b]$. Then it follows from [3] that

$$
u \in \mathrm{AC}[a, b] \quad \text { if and only if } \quad u(x)=u(a)+\int_{a}^{x} v(t) d t, \quad v(t) \in L[a, b]
$$

Obviously,

$$
\mathrm{AC}[a, b] \subset X_{\mu}[a, b]
$$

The latter is a Banach space under its norm. We further define the space

$$
\operatorname{AC}_{0}[a, b]=\left\{u: u(x) \in \mathrm{AC}[a, b] \text { with } u(a)=0 \text { and }\|u\|_{0}=\int_{a}^{b}\left|u^{\prime}(x)\right| d x<\infty\right\}
$$

Clearly, $\|u\|_{0}$ is a norm in $\mathrm{AC}_{0}[a, b]$. Indeed, if $\|u\|_{0}=0$ then $u(x)=u(a)=0$. To show that $\mathrm{AC}_{0}[a, b]$ is complete, we assume $\left\{u_{n}(x)\right\}$ is a Cauchy sequence in $\mathrm{AC}_{0}[a, b]$, then we need to find a function $u(x)$ such that $u(x)$ is absolutely continuous and $u_{n} \rightarrow u$ under its norm. Since $\left\{u_{n}(x)\right\}$ is Cauchy in $\mathrm{AC}_{0}[a, b]$, we claim that $u_{n}(a)=0$ and $\left\{u_{n}^{\prime}(x)\right\}$ is Cauchy in $L[a, b]$. Hence, there exists $g \in L[a, b]$ such that $u_{n}^{\prime} \rightarrow g$ in $L[a, b]$. Define

$$
u(x)=\int_{a}^{x} g(\tau) d \tau
$$

Then $u(a)=0$ and $u(x)$ is absolutely continuous on $[a, b]$, and

$$
\left\|u_{n}-u\right\|_{0} \leq \int_{a}^{b}\left|u_{n}^{\prime}(x)-g(\tau)\right| d \tau
$$

converges to zero. Therefore, $\mathrm{AC}_{0}[a, b]$ is a Banach space. 
Lemma 1.1 If $\alpha>0, \mu \in R$, and $0<a<b<\infty$, then the operator $\mathcal{J}_{a+, \mu}^{\alpha}$ is bounded in $\mathrm{AC}_{0}[a, b]$ and

$$
\left\|\mathcal{J}_{a+, \mu}^{\alpha} u\right\|_{0} \leq \frac{C_{\mu}}{\Gamma(\alpha+1)}\left[\log \left(\frac{b}{a}\right)\right]^{\alpha}\|u\|_{0}
$$

where $C_{\mu}$ is the maximum value of the function

$$
w(t, x)=\left(\frac{t}{x}\right)^{\mu}
$$

on $[a, b] \times[a, b]$.

Proof Let $u \in \mathrm{AC}_{0}[a, b]$. Then

$$
u(t)=\int_{a}^{t} v(s) d s=\int_{a}^{t} u^{\prime}(s) d s, \quad v(s)=u^{\prime}(s) \in L[a, b],
$$

and

$$
\begin{aligned}
\mathcal{J}_{a+, \mu}^{\alpha} u & =\mathcal{J}_{a+, \mu}^{\alpha} \int_{a}^{t} v(s) d s=\frac{1}{\Gamma(\alpha)} \int_{a}^{x}\left(\frac{t}{x}\right)^{\mu}\left(\log \frac{x}{t}\right)^{\alpha-1} \int_{a}^{t} v(s) d s \frac{d t}{t} \\
& =\frac{1}{\Gamma(\alpha)} \int_{a}^{x} v(s) d s \int_{s}^{x}\left(\frac{t}{x}\right)^{\mu}\left(\log \frac{x}{t}\right)^{\alpha-1} \frac{d t}{t}
\end{aligned}
$$

by changing the order of integration. Using

$$
0 \leq\left(\frac{t}{x}\right)^{\mu} \leq C_{\mu}
$$

we imply that

$$
\begin{aligned}
& \left|v(s) \int_{s}^{x}\left(\frac{t}{x}\right)^{\mu}\left(\log \frac{x}{t}\right)^{\alpha-1} \frac{d t}{t}\right| \leq \frac{C_{\mu}}{\alpha}|v(s)|\left[\log \left(\frac{b}{a}\right)\right]^{\alpha} \in L[a, b], \quad \text { and } \\
& \left\|\mathcal{J}_{a+, \mu}^{\alpha} u\right\|_{0} \leq \frac{C_{\mu}}{\Gamma(\alpha+1)}\left[\log \left(\frac{b}{a}\right)\right]^{\alpha}\|u\|_{0} .
\end{aligned}
$$

This completes the proof of Lemma 1.1.

Kilbas showed the following lemma in reference [2], which is soon to be used.

\section{Lemma 1.2}

(i) If $\alpha>0, \beta>0, \mu \in R$, and $u \in X_{\mu}[a, b]$, then the semigroup property holds

$$
\mathcal{J}_{a+, \mu}^{\alpha} \mathcal{J}_{a+, \mu}^{\beta} u=\mathcal{J}_{a+, \mu}^{\alpha+\beta} u
$$

(ii) If $0<\alpha<1$ and $u \in \mathrm{AC}[a, b]$, then

$$
\mathcal{J}_{a+, \mu}^{\alpha} \mathcal{D}_{a+, \mu}^{\alpha} u=u
$$


Let $u \in \mathrm{AC}[a, b]$ and $0<\beta<1$. It follows from Lemma 1.2 that

$$
\mathcal{J}_{a+, \mu}^{\alpha} \mathcal{D}_{a+, \mu}^{\beta} u=\mathcal{J}_{a+, \mu}^{\alpha-\beta} u
$$

if $\alpha \geq \beta$.

Let $0<\alpha_{0}<\alpha_{1}<\cdots<\alpha_{n}<1$ and $0 \leq \beta_{n+1}<\cdots<\beta_{m} \in R$, where $n=0,1, \ldots$ and $m>n$. In this paper, we show the uniqueness of solutions for the following new nonlinear Hadamard-type integro-differential equation for all $\mu \in R$ in the space $\mathrm{AC}_{0}[a, b]$ :

$$
\begin{aligned}
& \mathcal{D}_{a+, \mu}^{\alpha_{n}} u+a_{n-1} \mathcal{D}_{a+, \mu}^{\alpha_{n-1}} u+\cdots+a_{0} \mathcal{D}_{a+, \mu}^{\alpha_{0}} u+b_{n+1} \mathcal{J}_{a+, \mu}^{\beta_{n+1}} u+\cdots+b_{m} \mathcal{J}_{a+, \mu}^{\beta_{m}} u \\
& \quad=\int_{a}^{x} f\left(\tau, u^{\prime}(\tau)\right) d \tau
\end{aligned}
$$

by Banach's contraction principle and Babenko's approach [4], with two applicable examples presented to illustrate the main results. It seems impossible to obtain these by any existing integral transforms or analytic local model methods. Babenko's approach treats integral operators like variables in solving differential and integral equations. The method itself is close to the Laplace transform method in the ordinary sense, but it can be used in more cases $[5,6]$, such as dealing with integral or fractional differential equations with distributions whose Laplace transforms do not exist in the classical sense. Furthermore, it works well on certain differential or integral equations whose solutions cannot be achieved by the local model. Clearly, it is always necessary to show convergence of the series obtained as solutions. Recently, Li studied the generalized Abel's integral equations of the first [7] and second kind with variable coefficients by Babenko's technique [8-10].

It is well known that fractional calculus $[3,11,12]$ has been an emergent tool which uses fractional differential and integral equations to develop more sophisticated mathematical models that can accurately describe complex systems. There are many definitions of fractional derivatives available in the literature, such as the Riemann-Liouville derivative which played an important role in the development of the theory of fractional analysis. However, the commonly used is the Hadamard fractional derivative (with $\mu=0$ ) given by Hadamard in [13]. Butzer et al. [14-16] studied various properties of the Hadamardtype derivative which is more generalized than the Hadamard fractional derivative. In particular, Hadamard fractional differential equations with boundary value problems or initial conditions have been investigated by researchers using fixed point theories [17, 18]. In 2014, Thiramanus et al. [19] studied the existence and uniqueness of solutions for a fractional boundary value problem involving Hadamard differential equations of order $q \in(1,2]$ and nonlocal fractional integral boundary conditions by fixed point theories. In 2018, Matar [20] obtained the solution of the linear equations with the initial conditions (three terms on the left-hand side at most and a given function on the right) by the parameter technique, and then investigated the existence problems of the corresponding nonlinear types of Hadamard equations using fixed point theorems. Very recently, Ding et al. [21] applied the fixed point index and nonnegative matrices to study the existence of positive solutions for a system of Hadamard-type fractional differential equations with semipositone nonlinearities. In 1967, Caputo [22] introduced another type of fractional derivative which has an advantage over $\mathrm{R}-\mathrm{L}$ derivative in differential equations since it does not require to define fractional order initial conditions. Jarad et al. [23] defined the 
Caputo-type modification of the Hadamard fractional derivatives which preserve physically interpretable initial conditions similar to the ones in Caputo fractional derivatives. Gambo et al. [24] further presented the generalization of the fundamental theorem of fractional calculus (FTFC) in the Caputo-Hadamard setting with several new results. Adjabi et al. [25] studied Cauchy problems for a differential equation with a left CaputoHadamard fractional derivative in spaces of continuously differentiable functions.

There are new studies on fixed point theorems for different operators on metric spaces [26-28], as well as their applications in differential and integral equations, existence and uniqueness of solutions for equations [29-31]. Palve et al. [32] recently constructed the existence and uniqueness of solutions for the fractional implicit differential equation with boundary condition of the form

$$
\begin{aligned}
& { }_{H} D_{1+}^{\alpha, \beta} u(x)=f\left(x, u(x),{ }_{H} D_{1+}^{\alpha, \beta} u(x)\right), \quad 0<\alpha<1,0 \leq \beta \leq 1, x \in[1, b] \\
& \mathcal{J}_{1+, 0}^{1-\gamma} c_{1} u(x)+c_{2} u\left(b^{-}\right)=c_{3}, \quad \alpha \leq \gamma=\alpha+\beta(1-\alpha)
\end{aligned}
$$

where ${ }_{H} D_{1+}^{\alpha, \beta}$ is the Hilfer-Hadamard type fractional derivative of order $\alpha$ and type $\beta$ given by

$$
{ }_{H} D_{1+}^{\alpha, \beta}=\mathcal{J}_{1+, 0}^{\beta(n-\alpha)} D^{n} \mathcal{J}_{1+, 0}^{(1-\beta)(n-\alpha)}, \quad n-1<\alpha<n,
$$

and $c_{1}, c_{2}, c_{3} \in R$ with $c_{1}+c_{2} \neq 0$ and $c_{2} \neq 0$. Li [33] obtained uniqueness of solutions for the coupled system of integral equations

$$
\left\{\begin{array}{l}
a_{n}\left(\mathcal{J}_{a+, \mu}^{\alpha_{n}} u\right)(x)+\cdots+a_{1}\left(\mathcal{J}_{a+, \mu}^{\alpha_{1}} u\right)(x)+u(x)=g_{1}(x, u(x), v(x)), \\
b_{n}\left(\mathcal{J}_{a^{+}, \mu}^{\beta_{n}} v\right)(x)+\cdots+b_{1}\left(\mathcal{J}_{a^{+}, \mu}^{\beta_{1}} v\right)(x)+v(x)=g_{2}(x, u(x), v(x)),
\end{array}\right.
$$

on the product space $X_{\mu}[a, b] \times X_{\mu}[a, b]$ (it is a Banach space), based on Babenko's approach and Banach's contraction principle.

\section{Main results}

Theorem 2.1 Assume that $a_{i}$ and $b_{j}$ for $i=0,1, \ldots, n-1$ and $j=n+1, \ldots, m$ are arbitrary complex numbers, and $g \in \mathrm{AC}_{0}[a, b]$. In addition, we let $0<\alpha_{0}<\alpha_{1}<\cdots<\alpha_{n}<1$ and $0 \leq \beta_{n+1}<\cdots<\beta_{m} \in R$, where $n=0,1, \ldots$ Then equation

$$
\mathcal{D}_{a+, \mu}^{\alpha_{n}} u+a_{n-1} \mathcal{D}_{a+, \mu}^{\alpha_{n-1}} u+\cdots+a_{0} \mathcal{D}_{a+, \mu}^{\alpha_{0}} u+b_{n+1} \mathcal{J}_{a+, \mu}^{\beta_{n+1}} u+\cdots+b_{m} \mathcal{J}_{a+, \mu}^{\beta_{m}} u=g(x)
$$

has a unique solution

$$
u(x)=\sum_{k=0}^{\infty}(-1)^{k} \sum_{k_{1}+\cdots+k_{m}=k}\left(\begin{array}{c}
k \\
k_{1}, k_{2}, \ldots, k_{m}
\end{array}\right) a_{n-1}^{k_{1}} \cdots b_{m}^{k_{m}} \mathcal{J}_{a+, \mu}^{k_{1}\left(\alpha_{n}-\alpha_{n-1}\right)+\cdots+k_{m}\left(\alpha_{n}+\beta_{m}\right)+\alpha_{n}} g
$$

in the space $\mathrm{AC}_{0}[a, b]$.

Proof Applying the operator $\mathcal{J}_{a+, \mu}^{\alpha_{n}}$ to both sides of equation (2), we get

$$
\begin{array}{r}
\mathcal{J}_{a+, \mu}^{\alpha_{n}} \mathcal{D}_{a+, \mu}^{\alpha_{n}} u+a_{n-1} \mathcal{J}_{a+, \mu}^{\alpha_{n}} \mathcal{D}_{a+, \mu}^{\alpha_{n-1}} u+\cdots+a_{0} \mathcal{J}_{a+, \mu}^{\alpha_{n}} \mathcal{D}_{a+, \mu}^{\alpha_{0}} u \\
+b_{n+1} \mathcal{J}_{a+, \mu}^{\alpha_{n}} \mathcal{J}_{a+, \mu}^{\beta_{n+1}} u+\cdots+b_{m} \mathcal{J}_{a+, \mu}^{\alpha_{n}} \mathcal{J}_{a+, \mu}^{\beta_{m}} u=\mathcal{J}_{a+, \mu}^{\alpha_{n}} g
\end{array}
$$


Using Lemma 1.2,

$$
\begin{aligned}
u+ & a_{n-1} \mathcal{J}_{a+, \mu}^{\alpha_{n}-\alpha_{n-1}} u+\cdots+a_{0} \mathcal{J}_{a+, \mu}^{\alpha_{n}-\alpha_{0}} u \\
& +b_{n+1} \mathcal{J}_{a+, \mu}^{\alpha_{n}+\beta_{n+1}} u+\cdots+b_{m} \mathcal{J}_{a+, \mu}^{\alpha_{n}+\beta_{m}} u=\mathcal{J}_{a+, \mu}^{\alpha_{n}} g
\end{aligned}
$$

by noting that $0<\alpha_{0}<\alpha_{1}<\cdots<\alpha_{n}<1$. Hence,

$$
\begin{aligned}
& \left(1+a_{n-1} \mathcal{J}_{a+, \mu}^{\alpha_{n}-\alpha_{n-1}}+\cdots+a_{0} \mathcal{J}_{a+, \mu}^{\alpha_{n}-\alpha_{0}}+b_{n+1} \mathcal{J}_{a+, \mu}^{\alpha_{n}+\beta_{n+1}}+\cdots+b_{m} \mathcal{J}_{a+, \mu}^{\alpha_{n}+\beta_{m}}\right) u \\
& \quad=\mathcal{J}_{a+, \mu}^{\alpha_{n}} g .
\end{aligned}
$$

By Babenko's method we come to

$$
\begin{aligned}
u(x) & =\left(1+a_{n-1} \mathcal{J}_{a+, \mu}^{\alpha_{n}-\alpha_{n-1}}+\cdots+b_{m} \mathcal{J}_{a+, \mu}^{\alpha_{n}+\beta_{m}}\right)^{-1} \mathcal{J}_{a+, \mu}^{\alpha_{n} g} \\
& =\sum_{k=0}^{\infty}(-1)^{k}\left(a_{n-1} \mathcal{J}_{a+, \mu}^{\alpha_{n}-\alpha_{n-1}}+\cdots+b_{m} \mathcal{J}_{a+, \mu}^{\alpha_{n}+\beta_{m}}\right)^{k} \mathcal{J}_{a+, \mu}^{\alpha_{n} g} \\
& =\sum_{k=0}^{\infty}(-1)^{k} \sum_{k_{1}+\cdots+k_{m}=k}\left(\begin{array}{c}
k \\
k_{1}, k_{2}, \ldots, k_{m}
\end{array}\right)\left(a_{n-1} \mathcal{J}_{a+, \mu}^{\alpha_{n}-\alpha_{n-1}}\right)^{k_{1}} \cdots\left(b_{m} \mathcal{J}_{a+, \mu}^{\alpha_{n}+\beta_{m}}\right)^{k_{m}} \mathcal{J}_{a+, \mu}^{\alpha_{n} g} g \\
& =\sum_{k=0}^{\infty}(-1)^{k} \sum_{k_{1}+\cdots+k_{m}=k}\left(\begin{array}{c}
k \\
k_{1}, k_{2}, \ldots, k_{m}
\end{array}\right) a_{n-1}^{k_{1}} \mathcal{J}_{a+, \mu}^{k_{1}\left(\alpha_{n}-\alpha_{n-1}\right)} \cdots b_{m}^{k_{m}} \mathcal{J}_{a+, \mu}^{k_{m}\left(\alpha_{n}+\beta_{m}\right)} \mathcal{J}_{a+, \mu}^{\alpha_{n} g} \\
& =\sum_{k=0}^{\infty}(-1)^{k} \sum_{k_{1}+\cdots+k_{m}=k}\left(\begin{array}{c}
k \\
k_{1}, k_{2}, \ldots, k_{m}
\end{array}\right) a_{n-1}^{k_{1}} \cdots b_{m}^{k_{m}} \mathcal{J}_{a+, \mu}^{k_{1}\left(\alpha_{n}-\alpha_{n-1}\right)+\cdots+k_{m}\left(\alpha_{n}+\beta_{m}\right)+\alpha_{n} g,}
\end{aligned}
$$

using Lemma 1.2 and the multinomial theorem. Clearly, $u(a)=0$ since $\alpha_{n}>0$ and

$$
\left(\mathcal{J}_{a+, \mu}^{k_{1}\left(\alpha_{n}-\alpha_{n-1}\right)+\cdots+k_{m}\left(\alpha_{n}+\beta_{m}\right)+\alpha_{n}} g\right)(a)=0 .
$$

It remains to show that the series converges in the space $\mathrm{AC}_{0}[a, b]$ and is absolutely continuous on $[a, b]$. By Lemma 1.1,

$$
\left\|\mathcal{J}_{a+, \mu}^{k_{1}\left(\alpha_{n}-\alpha_{n-1}\right)+\cdots+k_{m}\left(\alpha_{n}+\beta_{m}\right)+\alpha_{n}} g\right\|_{0} \leq K\|g\|_{0},
$$

where

$$
\begin{aligned}
K= & \frac{C_{\mu}}{\Gamma\left(k_{1}\left(\alpha_{n}-\alpha_{n-1}\right)+\cdots+k_{m}\left(\alpha_{n}+\beta_{m}\right)+\alpha_{n}+1\right)} \\
& \cdot\left(\log \frac{b}{a}\right)^{k_{1}\left(\alpha_{n}-\alpha_{n-1}\right)+\cdots+k_{m}\left(\alpha_{n}+\beta_{m}\right)+\alpha_{n}}
\end{aligned}
$$


Therefore,

$$
\begin{aligned}
\|u\|_{0} \leq & C_{\mu} \sum_{k=0}^{\infty} \sum_{k_{1}+\cdots+k_{m}=k}\left(\begin{array}{c}
k \\
k_{1}, k_{2}, \ldots, k_{m}
\end{array}\right) \\
& \cdot \frac{\left(\left|a_{n-1}\right|\left(\log \frac{b}{a}\right)^{\alpha_{n}-\alpha_{n-1}}\right)^{k_{1}} \ldots\left(\left|b_{m}\right|\left(\log \frac{b}{a}\right)^{\alpha_{n}+\beta_{m}}\right)^{k_{m}}}{\Gamma\left(k_{1}\left(\alpha_{n}-\alpha_{n-1}\right)+\cdots+k_{m}\left(\alpha_{n}+\beta_{m}\right)+\alpha_{n}+1\right)}\|g\|_{0} \\
= & C_{\mu} E_{\left(\alpha_{n}-\alpha_{n-1}, \ldots, \alpha_{n}+\beta_{m}, \alpha_{n}+1\right)}\left(\left|a_{n-1}\right|\left(\log \frac{b}{a}\right)^{\alpha_{n}-\alpha_{n-1}}, \ldots,\left|b_{m}\right|\left(\log \frac{b}{a}\right)^{\alpha_{n}+\beta_{m}}\right)\|g\|_{0},
\end{aligned}
$$

where

$$
E_{\left(\alpha_{n}-\alpha_{n-1}, \ldots, \alpha_{n}+\beta_{m}, \alpha_{n}+1\right)}\left(\left|a_{n-1}\right|\left(\log \frac{b}{a}\right)^{\alpha_{n}-\alpha_{n-1}}, \ldots,\left|b_{m}\right|\left(\log \frac{b}{a}\right)^{\alpha_{n}+\beta_{m}}\right)<\infty
$$

is the value at

$$
z_{1}=\left|a_{n-1}\right|\left(\log \frac{b}{a}\right)^{\alpha_{n}-\alpha_{n-1}}, \quad \ldots, \quad z_{m}=\left|b_{m}\right|\left(\log \frac{b}{a}\right)^{\alpha_{n}+\beta_{m}}
$$

of the multivariate Mittag-Leffler function $E_{\left(\alpha_{n}-\alpha_{n-1}, \ldots, \alpha_{n}+\beta_{m}, \alpha_{n}+1\right)}\left(z_{1}, \ldots, z_{m}\right)$ given in [12]. Thus, the series on the right-hand side of equation (3) is convergent. To see $u(x)$ is absolutely continuous,

$$
\begin{aligned}
u(x)= & \sum_{k=0}^{\infty}(-1)^{k} \sum_{k_{1}+\cdots+k_{m}=k}\left(\begin{array}{c}
k \\
k_{1}, k_{2}, \ldots, k_{m}
\end{array}\right) a_{n-1}^{k_{1}} \cdots b_{m}^{k_{m}} \\
& \cdot \mathcal{J}_{a+, \mu}^{k_{1}\left(\alpha_{n}-\alpha_{n-1}\right)+\cdots+k_{m}\left(\alpha_{n}+\beta_{m}\right)+\alpha_{n}} \int_{a}^{t} g^{\prime}(s) d s \\
= & \sum_{k=0}^{\infty}(-1)^{k} \sum_{k_{1}+\cdots+k_{m}=k}\left(\begin{array}{c}
k \\
k_{1}, k_{2}, \ldots, k_{m}
\end{array}\right) a_{n-1}^{k_{1}} \cdots b_{m}^{k_{m}} \\
& \cdot \frac{1}{\Gamma\left(k_{1}\left(\alpha_{n}-\alpha_{n-1}\right)+\cdots+k_{m}\left(\alpha_{n}+\beta_{m}\right)+\alpha_{n}\right)} \int_{a}^{x} g^{\prime}(s) d s \\
& \cdot \int_{s}^{x}\left(\frac{t}{x}\right)^{\mu}\left(\log \frac{x}{t}\right)^{k_{1}\left(\alpha_{n}-\alpha_{n-1}\right)+\cdots+k_{m}\left(\alpha_{n}+\beta_{m}\right)+\alpha_{n}-1} \frac{d t}{t} \\
= & \int_{a}^{x} \sum_{k=0}^{\infty}(-1)^{k} \sum_{k_{1}+\cdots+k_{m}=k}\left(\begin{array}{c}
k \\
k_{1}, k_{2}, \ldots, k_{m}
\end{array}\right) a_{n-1}^{k_{1}} \cdots b_{m}^{k_{m}} \\
& \cdot \frac{g^{\prime}(s)}{\Gamma\left(k_{1}\left(\alpha_{n}-\alpha_{n-1}\right)+\cdots+k_{m}\left(\alpha_{n}+\beta_{m}\right)+\alpha_{n}\right)} \\
& \cdot \int_{s}^{x}\left(\frac{t}{x}\right)^{\mu}\left(\log \frac{x}{t}\right)^{k_{1}\left(\alpha_{n}-\alpha_{n-1}\right)+\cdots+k_{m}\left(\alpha_{n}+\beta_{m}\right)+\alpha_{n}-1} \frac{d t}{t} d s,
\end{aligned}
$$


as the function inside of the outer integral

$$
\begin{gathered}
\sum_{k=0}^{\infty}(-1)^{k} \sum_{k_{1}+\cdots+k_{m}=k}\left(\begin{array}{c}
k \\
k_{1}, k_{2}, \ldots, k_{m}
\end{array}\right) a_{n-1}^{k_{1}} \cdots b_{m}^{k_{m}} \\
\cdot \frac{g^{\prime}(s)}{\Gamma\left(k_{1}\left(\alpha_{n}-\alpha_{n-1}\right)+\cdots+k_{m}\left(\alpha_{n}+\beta_{m}\right)+\alpha_{n}\right)} \\
\cdot \int_{s}^{x}\left(\frac{t}{x}\right)^{\mu}\left(\log \frac{x}{t}\right)^{k_{1}\left(\alpha_{n}-\alpha_{n-1}\right)+\cdots+k_{m}\left(\alpha_{n}+\beta_{m}\right)+\alpha_{n}-1} \frac{d t}{t}
\end{gathered}
$$

uniformly converges with respect to $t$ and belongs to $L[a, b]$ from Lemma 1.1 and the multivariate Mittag-Leffler function used above. Thus, $u(x)$ is absolutely continuous on $[a, b]$. To verify that the obtained series is a solution, we substitute it into the left-hand side of equation (2):

$$
\begin{aligned}
& \mathcal{D}_{a+, \mu}^{\alpha_{n}}\left(\sum_{k=0}^{\infty}(-1)^{k} \sum_{k_{1}+\cdots+k_{m}=k}\left(\begin{array}{c}
k \\
k_{1}, k_{2}, \ldots, k_{m}
\end{array}\right) a_{n-1}^{k_{1}} \cdots b_{m}^{k_{m}}\right. \\
& \left.\cdot \mathcal{J}_{a+, \mu}^{k_{1}\left(\alpha_{n}-\alpha_{n-1}\right)+\cdots+k_{m}\left(\alpha_{n}+\beta_{m}\right)+\alpha_{n}} g\right) \\
& +\left(\sum_{k=0}^{\infty}(-1)^{k} \sum_{k_{1}+\cdots+k_{m}=k}\left(\begin{array}{c}
k \\
k_{1}, k_{2}, \ldots, k_{m}
\end{array}\right) a_{n-1}^{k_{1}+1} \cdots b_{m}^{k_{m}}\right. \\
& \left.\cdot \mathcal{J}_{a+, \mu}^{\left(k_{1}+1\right)\left(\alpha_{n}-\alpha_{n-1}\right)+\cdots+k_{m}\left(\alpha_{n}+\beta_{m}\right)} g\right) \\
& +\cdots+\left(\sum_{k=0}^{\infty}(-1)^{k} \sum_{k_{1}+\cdots+k_{m}=k}\left(\begin{array}{c}
k \\
k_{1}, k_{2}, \ldots, k_{m}
\end{array}\right) a_{n-1}^{k_{1}} \cdots b_{m}^{k_{m}+1}\right. \\
& \left.\cdot \mathcal{J}_{a+, \mu}^{k_{1}\left(\alpha_{n}-\alpha_{n-1}\right)+\cdots+\left(k_{m}+1\right)\left(\alpha_{n}+\beta_{m}\right)} g\right) \\
& =\mathcal{D}_{a+, \mu}^{\alpha_{n}}\left(\mathcal{J}_{a+, \mu}^{\alpha_{n}} g+\sum_{k=1}^{\infty}(-1)^{k} \sum_{k_{1}+\cdots+k_{m}=k}\left(\begin{array}{c}
k \\
k_{1}, k_{2}, \ldots, k_{m}
\end{array}\right) a_{n-1}^{k_{1}} \cdots b_{m}^{k_{m}}\right. \\
& \left.\cdot \mathcal{J}_{a+, \mu}^{k_{1}\left(\alpha_{n}-\alpha_{n-1}\right)+\cdots+k_{m}\left(\alpha_{n}+\beta_{m}\right)+\alpha_{n}} g\right) \\
& +\left(\sum_{k=0}^{\infty}(-1)^{k} \sum_{k_{1}+\cdots+k_{m}=k}\left(\begin{array}{c}
k \\
k_{1}, k_{2}, \ldots, k_{m}
\end{array}\right) a_{n-1}^{k_{1}+1} \cdots b_{m}^{k_{m}}\right. \\
& \left.\cdot \mathcal{J}_{a+, \mu}^{\left(k_{1}+1\right)\left(\alpha_{n}-\alpha_{n-1}\right)+\cdots+k_{m}\left(\alpha_{n}+\beta_{m}\right)} g\right) \\
& +\cdots+\left(\sum_{k=0}^{\infty}(-1)^{k} \sum_{k_{1}+\cdots+k_{m}=k}\left(\begin{array}{c}
k \\
k_{1}, k_{2}, \ldots, k_{m}
\end{array}\right) a_{n-1}^{k_{1}} \cdots b_{m}^{k_{m}+1}\right. \\
& \left.\cdot \mathcal{J}_{a+, \mu}^{k_{1}\left(\alpha_{n}-\alpha_{n-1}\right)+\cdots+\left(k_{m}+1\right)\left(\alpha_{n}+\beta_{m}\right)} g\right)
\end{aligned}
$$




$$
\begin{aligned}
= & +\left(\sum_{k=1}^{\infty}(-1)^{k} \sum_{k_{1}+\cdots+k_{m}=k}\left(\begin{array}{c}
k \\
k_{1}, k_{2}, \ldots, k_{m}
\end{array}\right) a_{n-1}^{k_{1}} \cdots b_{m}^{k_{m}}\right. \\
& \cdot \mathcal{J}_{a+, \mu}^{\left.k_{1}\left(\alpha_{n}-\alpha_{n-1}\right)+\cdots+k_{m}\left(\alpha_{n}+\beta_{m}\right)_{g}\right)} \\
& +\left(\sum_{k=0}^{\infty}(-1)^{k} \sum_{k_{1}+\cdots+k_{m}=k}\left(\begin{array}{c}
k \\
k_{1}, k_{2}, \ldots, k_{m}
\end{array}\right) a_{n-1}^{k_{1}+1} \cdots b_{m}^{k_{m}}\right. \\
& \left.\cdot \mathcal{J}_{a+, \mu}^{\left(k_{1}+1\right)\left(\alpha_{n}-\alpha_{n-1}\right)+\cdots+k_{m}\left(\alpha_{n}+\beta_{m}\right)} g\right) \\
& +\cdots+\left(\sum_{k=0}^{\infty}(-1)^{k} \sum_{k_{1}+\cdots+k_{m}=k}\left(k_{1}, k_{2}, \ldots, k_{m}\right) a_{n-1}^{k_{1}} \cdots b_{m}^{k_{m}+1}\right. \\
& \left.\cdot \mathcal{J}_{a+, \mu}^{k_{1}\left(\alpha_{n}-\alpha_{n-1}\right)+\cdots+\left(k_{m}+1\right)\left(\alpha_{n}+\beta_{m}\right)} g\right)=g
\end{aligned}
$$

by the cancelation. Note that all series are absolutely convergent and the term rearrangements are feasible for the cancelation.

Indeed,

$$
\begin{aligned}
& -\sum_{k_{1}+\cdots+k_{m}=1}\left(\begin{array}{c}
k \\
k_{1}, k_{2}, \ldots, k_{m}
\end{array}\right) a_{n-1}^{k_{1}} \cdots b_{m}^{k_{m}} \mathcal{J}_{a+, \mu}^{k_{1}\left(\alpha_{n}-\alpha_{n-1}\right)+\cdots+k_{m}\left(\alpha_{n}+\beta_{m}\right)} g \\
& \quad+\sum_{k_{1}+\cdots+k_{m}=0}\left(\begin{array}{c}
k \\
k_{1}, k_{2}, \ldots, k_{m}
\end{array}\right) a_{n-1}^{k_{1}+1} \cdots b_{m}^{k_{m}} \mathcal{J}_{a+, \mu}^{\left(k_{1}+1\right)\left(\alpha_{n}-\alpha_{n-1}\right)+\cdots+k_{m}\left(\alpha_{n}+\beta_{m}\right)} g \\
& \quad+\cdots+\sum_{k_{1}+\cdots+k_{m}=0}\left(\begin{array}{c}
k \\
k_{1}, k_{2}, \ldots, k_{m}
\end{array}\right) a_{n-1}^{k_{1}} \cdots b_{m}^{k_{m}+1} \mathcal{J}_{a+, \mu}^{k_{1}\left(\alpha_{n}-\alpha_{n-1}\right)+\cdots+\left(k_{m}+1\right)\left(\alpha_{n}+\beta_{m}\right)} g \\
& =0 .
\end{aligned}
$$

The rest terms cancel each other similarly.

Clearly, the uniqueness follows immediately from the fact that the integro-differential equation

$$
\mathcal{D}_{a+, \mu}^{\alpha_{n}} u+a_{n-1} \mathcal{D}_{a+, \mu}^{\alpha_{n-1}} u+\cdots+a_{0} \mathcal{D}_{a+, \mu}^{\alpha_{0}} u+b_{n+1} \mathcal{J}_{a+, \mu}^{\beta_{n+1}} u+\cdots+b_{m} \mathcal{J}_{a+, \mu}^{\beta_{m}} u=0
$$

only has solution zero by Babenko's method. This completes the proof of Theorem 2.1.

\section{Remark 1}

(i) It follows from Theorem 5.3 in [2] that for $0<\alpha<1$

$$
\left(\mathcal{D}_{a+, \mu}^{\alpha} u\right)(x)=\frac{x^{-\mu}}{\Gamma(1-\alpha)}\left[u_{0}(a)\left(\log \frac{x}{a}\right)^{-\alpha}+\int_{a}^{x}\left(\log \frac{x}{t}\right)^{-\alpha} u_{0}^{\prime}(t) d t\right]
$$

where $u_{0}(x)=x^{\mu} u(x) \in \mathrm{AC}[a, b]$. Hence, for $u \in \mathrm{AC}_{0}[a, b]$,

$$
\left(\mathcal{D}_{a+, \mu}^{\alpha} u\right)(x)=\frac{x^{-\mu}}{\Gamma(1-\alpha)} \int_{a}^{x}\left(\log \frac{x}{t}\right)^{-\alpha} u_{0}^{\prime}(t) d t, \quad \text { and }
$$




$$
\left(\mathcal{D}_{a+, \mu}^{\alpha} u\right)(a)=0
$$

(ii) A solution of equation (2) in the space $\mathrm{AC}_{0}[a, b]$ is said to be stable if $\forall \epsilon>0 \exists \delta>0$, such that $\|u\|_{0}<\epsilon$ if $\|g\|_{0}<\delta$. Using the inequality

$$
\begin{aligned}
\|u\|_{0} \leq & C_{\mu} E_{\left(\alpha_{n}-\alpha_{n-1}, \ldots, \alpha_{n}+\beta_{m}, \alpha_{n}+1\right)} \\
& \cdot\left(\left|a_{n-1}\right|\left(\log \frac{b}{a}\right)^{\alpha_{n}-\alpha_{n-1}}, \ldots,\left|b_{m}\right|\left(\log \frac{b}{a}\right)^{\alpha_{n}+\beta_{m}}\right)\|g\|_{0},
\end{aligned}
$$

we imply that the solution $u$ is stable.

(iii) The multivariate Mittag-Leffler function was initially introduced by Hadid and Luchko [34], who used it for solving linear fractional differential equations with constant coefficients by the operational method. Suthar et al. [35] studied some properties of generalized multivariate Mittag-Leffler function and established two theorems giving the image of this function under certain integral operators. Haubold et al. [36] presented a good survey of the Mittag-Leffler function, generalized Mittag-Leffler functions, Mittag-Leffler type functions, their interesting and useful properties, and applications in certain areas of physical and applied sciences. The Mittag-Leffler function plays an important role in the investigations of the fractional generalization of the kinetic equation, random walks, Lévy flights, superdiffusive transport and in the study of complex models.

Let $v>0$ and $x \geq 0$. The incomplete gamma function is defined by

$$
\gamma(\nu, x)=\int_{0}^{x} t^{\nu-1} e^{-t} d t
$$

From the recurrence relation [37]

$$
\gamma(\nu+1, x)=v \gamma(\nu, x)-x^{v} e^{-x},
$$

we get

$$
\gamma(v, x)=x^{\nu} \Gamma(v) e^{-x} \sum_{j=0}^{\infty} \frac{x^{j}}{\Gamma(v+j+1)} .
$$

Example 1 Let $0<a<x<b$. Then the Hadamard-type integro-differential equation

$$
\left(\mathcal{D}_{a+,-1}^{0.8} u\right)(x)+\left(\mathcal{D}_{a+,-1}^{0.7} u\right)(x)+\left(\mathcal{D}_{a+,-1}^{0.1} u\right)(x)+2\left(\mathcal{J}_{a+,-1}^{0.2} u\right)(x)-\left(\mathcal{J}_{a+,-1} u\right)(x)=x^{2},
$$

has the solution

$$
\begin{aligned}
u(x)= & a x \sum_{k=0}^{\infty}(-1)^{k} \sum_{k_{1}+k_{2}+k_{3}+k_{4}=k}\left(\begin{array}{c}
k \\
k_{1}, k_{2}, k_{3}, k_{4}
\end{array}\right) 2^{k_{3}}(-1)^{k_{4}} \\
& \cdot \sum_{j=0}^{\infty} \frac{(\log x / a)^{j+0.1 k_{1}+0.7 k_{2}+k_{3}+1.8 k_{4}+0.8}}{\Gamma\left(0.1 k_{1}+0.7 k_{2}+k_{3}+1.8 k_{4}+0.8+j+1\right)}
\end{aligned}
$$


in the space $\mathrm{AC}_{0}[a, b]$. Indeed, it follows from Lemma 2.4 in [2] that

$$
\left(\mathcal{J}_{a+, \mu}^{\alpha} t^{w}\right)(x)=\frac{\gamma(\alpha,(\mu+w) \log (x / a))}{\Gamma(\alpha)}(\mu+w)^{-\alpha} x^{w},
$$

where $\mu+w>0$.

By Theorem 2.1,

$$
\begin{aligned}
u(x)= & \sum_{k=0}^{\infty}(-1)^{k} \sum_{k_{1}+k_{2}+k_{3}+k_{4}=k}\left(\begin{array}{c}
k \\
k_{1}, k_{2}, k_{3}, k_{4}
\end{array}\right) 2^{k_{3}}(-1)^{k_{4}} \\
& \cdot\left(\mathcal{J}_{a+,-1}^{0.1 k_{1}+0.7 k_{2}+k_{3}+1.8 k_{4}+0.8} t^{2}\right)(x) \\
= & \sum_{k=0}^{\infty}(-1)^{k} \sum_{k_{1}+k_{2}+k_{3}+k_{4}=k}\left(\begin{array}{c}
k \\
k_{1}, k_{2}, k_{3}, k_{4}
\end{array}\right) 2^{k_{3}}(-1)^{k_{4}} \\
& \cdot \frac{\gamma\left(0.1 k_{1}+0.7 k_{2}+k_{3}+1.8 k_{4}+0.8, \log (x / a)\right)}{\Gamma\left(0.1 k_{1}+0.7 k_{2}+k_{3}+1.8 k_{4}+0.8\right)} x^{2} .
\end{aligned}
$$

Applying equation (5),

$$
\begin{aligned}
\gamma & \left(0.1 k_{1}+0.7 k_{2}+k_{3}+1.8 k_{4}+0.8, \log (x / a)\right) \\
= & (\log x / a)^{0.1 k_{1}+0.7 k_{2}+k_{3}+1.8 k_{4}+0.8} \Gamma\left(0.1 k_{1}+0.7 k_{2}+k_{3}+1.8 k_{4}+0.8\right) \\
& \cdot \frac{a}{x} \sum_{j=0}^{\infty} \frac{(\log x / a)^{j}}{\Gamma\left(0.1 k_{1}+0.7 k_{2}+k_{3}+1.8 k_{4}+0.8+j+1\right)} .
\end{aligned}
$$

Thus,

$$
\begin{aligned}
u(x)= & a x \sum_{k=0}^{\infty}(-1)^{k} \sum_{k_{1}+k_{2}+k_{3}+k_{4}=k}\left(\begin{array}{c}
k \\
k_{1}, k_{2}, k_{3}, k_{4}
\end{array}\right) 2^{k_{3}}(-1)^{k_{4}} \\
& \cdot \sum_{j=0}^{\infty} \frac{(\log x / a)^{j+0.1 k_{1}+0.7 k_{2}+k_{3}+1.8 k_{4}+0.8}}{\Gamma\left(0.1 k_{1}+0.7 k_{2}+k_{3}+1.8 k_{4}+0.8+j+1\right)}
\end{aligned}
$$

is the solution in the space $\mathrm{AC}_{0}[a, b]$.

The following theorem shows the uniqueness of equation (1).

Theorem 2.2 Assume that $f:[a, b] \times R \rightarrow R$ is a continuous function, and there exists a constant $C$ such that

$$
\left|f\left(x, y_{1}\right)-f\left(x, y_{2}\right)\right| \leq C\left|y_{1}-y_{2}\right|
$$

for all $x \in[a, b]$ and $y_{1}, y_{2} \in R$. Furthermore,

$$
C_{\mu} C E_{\left(\alpha_{n}-\alpha_{n-1}, \ldots, \alpha_{n}+\beta_{m}, \alpha_{n}+1\right)}\left(\left|a_{n-1}\right|\left(\log \frac{b}{a}\right)^{\alpha_{n}-\alpha_{n-1}}, \ldots,\left|b_{m}\right|\left(\log \frac{b}{a}\right)^{\alpha_{n}+\beta_{m}}\right)<1 .
$$

Then equation (1) has a unique solution in the space $\mathrm{AC}_{0}[a, b]$ for every $\mu \in R$. 
Proof Let $u \in \mathrm{AC}_{0}[a, b]$. Then

$$
\int_{a}^{x} f\left(\tau, u^{\prime}(\tau)\right) d \tau \in \mathrm{AC}_{0}[a, b]
$$

as $u^{\prime}(\tau) \in L[a, b]$ and $f\left(\tau, u^{\prime}(\tau)\right) \in L[a, b]$. Clearly,

$$
\begin{aligned}
\left\|\int_{a}^{x} f\left(\tau, u^{\prime}(\tau)\right) d \tau\right\|_{0} & =\int_{a}^{b}\left|f\left(x, u^{\prime}(x)\right)\right| d x \\
& \leq \int_{a}^{b}\left|f\left(x, u^{\prime}(x)\right)-f(x, 0)\right| d x+\int_{a}^{b}|f(x, 0)| d x \\
& \leq C \int_{a}^{b}\left|u^{\prime}(x)\right| d x+\int_{a}^{b}|f(x, 0)| d x<\infty .
\end{aligned}
$$

Define a mapping $T$ on $\mathrm{AC}_{0}[a, b]$ by

$$
\begin{aligned}
T(u)= & \sum_{k=0}^{\infty}(-1)^{k} \sum_{k_{1}+\cdots+k_{m}=k}\left(\begin{array}{c}
k \\
k_{1}, k_{2}, \ldots, k_{m}
\end{array}\right) a_{n-1}^{k_{1}} \cdots b_{m}^{k_{m}} \\
& \cdot \mathcal{J}_{a+, \mu}^{k_{1}\left(\alpha_{n}-\alpha_{n-1}\right)+\cdots+k_{m}\left(\alpha_{n}+\beta_{m}\right)+\alpha_{n}} \int_{a}^{t} f\left(\tau, u^{\prime}(\tau)\right) d \tau .
\end{aligned}
$$

Using inequality (4), we claim that

$$
\|T(u)\|_{0}<\infty \quad \text { and } \quad T(u)(a)=0 .
$$

Furthermore, $T(u)$ is absolutely continuous on $[a, b]$ from the proof of Theorem 2.1. Hence, $T$ is a mapping from $\mathrm{AC}_{0}[a, b]$ to $\mathrm{AC}_{0}[a, b]$. It remains to prove that $T$ is contractive. Indeed,

$$
\begin{aligned}
& \|T(u)-T(v)\|_{0} \\
& \leq C_{\mu} E_{\left(\alpha_{n}-\alpha_{n-1}, \ldots, \alpha_{n}+\beta_{m}, \alpha_{n}+1\right)}\left(\left|a_{n-1}\right|\left(\log \frac{b}{a}\right)^{\alpha_{n}-\alpha_{n-1}}, \ldots,\left|b_{m}\right|\left(\log \frac{b}{a}\right)^{\alpha_{n}+\beta_{m}}\right) \\
& \quad .\left\|\int_{a}^{t} f\left(\tau, u^{\prime}(\tau)\right) d \tau-\int_{a}^{t} f\left(\tau, v^{\prime}(\tau)\right) d \tau\right\|_{0}
\end{aligned}
$$

Since

$$
\begin{aligned}
\left\|\int_{a}^{t} f\left(\tau, u^{\prime}(\tau)\right) d \tau-\int_{a}^{t} f\left(\tau, v^{\prime}(\tau)\right) d \tau\right\|_{0} & =\int_{a}^{b}\left|f\left(t, u^{\prime}(t)\right)-f\left(t, v^{\prime}(t)\right)\right| d t \\
& \leq C \int_{a}^{b}\left|u^{\prime}(t)-v^{\prime}(t)\right| d t=C\|u-v\|_{0},
\end{aligned}
$$

we derive

$$
\begin{aligned}
& \|T(u)-T(v)\|_{0} \\
& \quad \leq C_{\mu} C E_{\left(\alpha_{n}-\alpha_{n-1}, \ldots, \alpha_{n}+\beta_{m}, \alpha_{n}+1\right)}\left(\left|a_{n-1}\right|\left(\log \frac{b}{a}\right)^{\alpha_{n}-\alpha_{n-1}}, \ldots,\left|b_{m}\right|\left(\log \frac{b}{a}\right)^{\alpha_{n}+\beta_{m}}\right)
\end{aligned}
$$




$$
\cdot\|u-v\|_{0}
$$

Therefore $T$ is contractive. This completes the proof of Theorem 2.2.

Example 2 Let $a=1, b=e$ and $\mu=2$. Then there is a unique solution for the following nonlinear Hadamard-type integro-differential equation:

$$
\begin{gathered}
\left(\mathcal{D}_{1+, 2}^{0.5} u\right)(x)+\left(\mathcal{J}_{1+, 2}^{0.5} u\right)(x)-\left(\mathcal{J}_{1+, 2}^{1.5} u\right)(x)+\left(\mathcal{J}_{1+, 2}^{2.1} u\right)(x) \\
=\int_{a}^{x}\left(\frac{t^{2}}{C\left(1+t^{100}\right)} \sin u^{\prime}(t)+\cos (\sin t)+e^{t^{2}}\right) d t
\end{gathered}
$$

where the constant $C$ is to be determined.

Clearly, $C_{2}=e^{2}$ is the maximum value of the function $\left(\frac{t}{x}\right)^{2}$ over the interval $[1, e] \times[1, e]$, and the function

$$
f(x, y)=\frac{x^{2}}{C\left(1+x^{100}\right)} \sin y+\cos (\sin x)+e^{x^{2}}
$$

is a continuous function from $[1, e] \times R$ to $R$ and satisfies

$$
\left|f\left(x, y_{1}\right)-f\left(x, y_{2}\right)\right| \leq \frac{x^{2}}{C\left(1+x^{100}\right)}\left|\sin y_{1}-\sin y_{2}\right| \leq \frac{x^{2}}{C\left(1+x^{100}\right)}\left|y_{1}-y_{2}\right| \leq \frac{1}{C}\left|y_{1}-y_{2}\right| .
$$

Obviously $\log b / a=1$. By Theorem 2.2, we need to calculate the value

$$
\begin{aligned}
& \sum_{k=0}^{\infty} \sum_{k_{1}+k_{2}+k_{3}=k}\left(\begin{array}{c}
k \\
k_{1}, k_{2}, k_{3}
\end{array}\right) \frac{1}{\Gamma\left(k_{1}+2 k_{2}+2.6 k_{2}+1.5\right)} \\
& =\sum_{k=0}^{\infty} \sum_{k_{1}+k_{2}+k_{3}=k}\left(\begin{array}{c}
k \\
k_{1}, k_{2}, k_{3}
\end{array}\right) \frac{1}{\Gamma\left(k+1.5+k_{2}+1.6 k_{3}\right)} \\
& =\frac{1}{\Gamma(1.5)}+\sum_{k=1}^{\infty} \sum_{k_{1}+k_{2}+k_{3}=k}\left(\begin{array}{c}
k \\
k_{1}, k_{2}, k_{3}
\end{array}\right) \frac{1}{\Gamma\left(k+1.5+k_{2}+1.6 k_{3}\right)} .
\end{aligned}
$$

For $k \geq 1$,

$$
\frac{1}{\Gamma\left(k+1.5+k_{2}+1.6 k_{3}\right)} \leq \frac{1}{\Gamma(k+1)}=\frac{1}{k !}, \quad \text { and } \quad \sum_{k_{1}+k_{2}+k_{3}=k}\left(\begin{array}{c}
k \\
k_{1}, k_{2}, k_{3}
\end{array}\right)=3^{k} .
$$

Therefore,

$$
\begin{aligned}
& \sum_{k=0}^{\infty} \sum_{k_{1}+k_{2}+k_{3}=k}\left(\begin{array}{c}
k \\
k_{1}, k_{2}, k_{3}
\end{array}\right) \frac{1}{\Gamma\left(k_{1}+2 k_{2}+2.6 k_{2}+1.5\right)} \leq \frac{1}{\Gamma(1.5)}+\sum_{k=1}^{\infty} \frac{3^{k}}{k !} \\
& <\frac{1}{2}+\sum_{k=0}^{\infty} \frac{3^{k}}{k !} .
\end{aligned}
$$

Then, choose a positive $C$ such that

$$
C e^{2}\left(\frac{1}{2}+\sum_{k=0}^{\infty} \frac{3^{k}}{k !}\right)<1
$$


By Theorem 2.2, equation (6) has a unique solution. We note that the series $\sum_{k=0}^{\infty} \frac{3^{k}}{k !}$ converges.

\section{Conclusions}

Using Babenko's approach and Banach's contraction principle, we have derived the uniqueness of solutions for the new nonlinear Hadamard-type integro-differential equation for all $\mu \in R$ :

$$
\begin{aligned}
& \mathcal{D}_{a+, \mu}^{\alpha_{n}} u+a_{n-1} \mathcal{D}_{a+, \mu}^{\alpha_{n-1}} u+\cdots+a_{0} \mathcal{D}_{a+, \mu}^{\alpha_{0}} u+b_{n+1} \mathcal{J}_{a+, \mu}^{\beta_{n+1}} u+\cdots+b_{m} \mathcal{J}_{a+, \mu}^{\beta_{m}} u \\
& \quad=\int_{a}^{x} f\left(\tau, u^{\prime}(\tau)\right) d \tau
\end{aligned}
$$

in the Banach space $\mathrm{AC}_{0}[a, b]$, with two examples given to illustrate the main theorems. The results obtained are fresh in the present studies, and they cannot be achieved via any existing integral transforms or local model methods to the best knowledge of the author.

\section{Acknowledgements}

The author is grateful to the three reviewers for their careful reading of the paper with productive comments and suggestions.

\section{Funding}

This work is supported by NSERC (Canada 2019-03907).

\section{Availability of data and materials}

Not applicable.

\section{Competing interests}

The author declares that they have no competing interests.

Authors' contributions

The author prepared, read, and approved the final manuscript.

\section{Publisher's Note}

Springer Nature remains neutral with regard to jurisdictional claims in published maps and institutional affiliations.

Received: 9 December 2020 Accepted: 24 February 2021 Published online: 15 March 2021

\section{References}

1. Kilbas, A.A.: Hadamard-type fractional calculus. J. Korean Math. Soc. 38, 1191-1204 (2001). https://doi.org/10.1016/j.bulsci.2011.12.004

2. Kilbas, A.A.: Hadamard-type integral equations and fractional calculus operators. Oper. Theory, Adv. Appl. 142, 175-188 (2003)

3. Samko, S.G., Kilbas, A.A., Marichev, O.I.: Fractional Integrals and Derivatives: Theory and Applications. Gordon \& Breach, New York (1993)

4. Babenkos, Y.l.: Heat and Mass Transfer. Khimiya, Leningrad (1986) (in Russian)

5. Podlubny, I.: Fractional Differential Equations. Academic Press, New York (1999)

6. Li, C., Clarkson, K.: Babenko's approach to Abel's integral equations. Mathematics (2018). https://doi.org/10.3390/math6030032

7. Li, C., Li, C.P., Clarkson, K.: Several results of fractional differential and integral equations in distribution. Mathematics (2018). https://doi.org/10.3390/math6060097

8. Li, C., Plowman, $\mathrm{H}$.: Solutions of the generalized Abel's integral equations of the second kind with variable coefficients. Axioms (2019). https://doi.org/10.3390/axioms8040137

9. Li, C.: The generalized Abel's integral equations on $R^{n}$ with variable coefficients. Fract. Differ. Calc. 10, 129-140 (2020)

10. Li, C., Huang, J.: Remarks on the linear fractional integro-differential equation with variable coefficients in distribution. Fract. Differ. Calc. 10, 57-77 (2020)

11. Gorenflo, R., Mainardi, F.: Fractional calculus: integral and differential equations of fractional order. In: Fractals and Fractional Calculus in Continuum Mechanics, pp. 223-276. Springer, New York (1997)

12. Kilbas, A.A., Srivastava, H.M., Trujillo, J.J.: Theory and Applications of Fractional Differential Equations. Elsevier, Amsterdam (2006)

13. Hadamard, J.: Essai sur l'etude des fonctions donnees par leur developpment de Taylor. J. Math. Pures Appl. 4 , 101-186 (1892) 
14. Butzer, P.L., Kilbas, A.A., Trujillo, J.J: Compositions of Hadamard-type fractional integration operators and the semigroup property. J. Math. Anal. Appl. 269, 387-400 (2002)

15. Butzer, P.L., Kilbas, A.A., Trujillo, J.J.: Fractional calculus in the Mellin setting and Hadamard-type fractional integrals, J. Math. Anal. Appl. 269, 1-27 (2002)

16. Butzer, P.L., Killbas, A.A., Trujillo, J.J: Mellin transform analysis and integration by parts for Hadamard-type fractional integrals. J. Math. Anal. Appl. 270, 1-15 (2002)

17. Ahmad, B., Ntouyas, S.K.: A fully Hadamard type integral boundary value problem of a coupled system of fractional differential equations. Fract. Calc. Appl. Anal. 17, 348-360 (2014)

18. Ahmad, B., Alsaedi, A., Ntouyas, S.K., Tariboon, J.: Hadamard-Type Fractional Differential Equations, Inclusions and Inequalities. Springer, Heidelberg (2017)

19. Thiramanus, P., Ntouyas, S.K., Tariboon, J.: Existence and uniqueness results for Hadamard-type fractional differential equations with nonlocal fractional integral boundary conditions. Abstr. Appl. Anal. 2014, Article ID 902054 (2014). https://doi.org/10.1155/2014/902054

20. Matar, M.M.: Solution of sequential Hadamard fractional differential equations by variation of parameter technique. Abstr. Appl. Anal. 2018, Article ID 9605353 (2018). https://doi.org/10.1155/2018/9605353

21. Ding, Y., Jiang, J., O'Regan, D., Xu, J.: Positive solutions for a system of Hadamard-type fractional differential equations with semipositone nonlinearities. Complexity 2020, Article ID 9742418 (2020). https://doi.org/10.1155/2020/9742418

22. Caputo, M.: Linear model of dissipation whose $Q$ is almost frequency independent. II. Geophys. J. Int. 13, 529-539 (1967)

23. Jarad, F., Abdeljawad, T., Baleanu, D.: Caputo-type modification of the Hadamard fractional derivatives. Adv. Differ. Equ. 2012, 142 (2012). http://www.advancesindifferenceequations.com/content/2012/1/142

24. Gambo, Y.Y., Jarad, F., Baleanu, D., Abdeljawad, T.: On Caputo modification of the Hadamard fractional derivatives. Adv. Differ. Equ. 2014, 10 (2014). http://www.advancesindifferenceequations.com/content/2014/1/10

25. Adjabi, Y., Jarad, F., Baleanu, D., Abdeljawad, T.: On Cauchy problems with Caputo Hadamard fractional derivatives. J. Comput. Anal. Appl. 21, 661-681 (2016)

26. Khan, A., Khan, H., Li, T., Akça, H., Khan, T.S.: Common fixed point theorems for weakly compatible self-mappings sustaining integral type contractions. Int. J. Appl. Math. Stat. 57, 43-55 (2018)

27. Khan, A., Abdeljawad, T., Shatanawi, W., Khan, H.: Fixed point theorems for quadruple self-mappings satisfying integral type inequalities. Filomat 34, 905-917 (2020)

28. Khan, A., Khan, H., Baleanu, D., Karapinar, E., Khan, T.S.: Fixed points of weakly compatible mappings satisfying a generalized common limit range property. J. Nonlinear Sci. Appl. 10, 5690-5700 (2017)

29. Abdo, M.S., Abdeljawad, T., Ali, S.M., Shah, K., Jarad, F.: Existence of positive solutions for weighted fractional order differential equations. Chaos Solitons Fractals 141, 110341 (2020)

30. Abdo, M.S.: Further results on the existence of solutions for generalized fractional quadratic functional integral equations. J. Math. Anal. Model. 1, 33-46 (2020). https://doi.org/10.48185/jmam.v111.2

31. Abdo, M.S., Panchal, S.K., Wahash, H.A.: Ulam-Hyers-Mittag-Leffler stability for a $\Psi$-Hilfer problem with fractional order and infinite delay. Res. Appl. Math. 7, 100115 (2020). https://doi.org/10.1016/j.rinam.2020.100115

32. Palve, L.A., Abdo, M.S., Panchal, S.K.: Some existence and stability results of Hilfer-Hadamard fractional implicit differential fractional equation in a weighted space. arXiv preprint (2019). arXiv:1910.08369

33. Li, C.: Uniqueness of the Hadamard-type integral equations. Adv. Differ. Equ. 2021, 40 (2021). https://doi.org/10.1186/s13662-020-03205-8

34. Hadid, S.B., Luchko, Y: An operational method for solving fractional differential equations of an arbitrary real order. Panam. Math. J. 6, 57-73 (1996)

35. Suthar, D.L., Andualem, M., Debalkie, B.: A study on generalized multivariable Mittag-Leffler function via generalized fractional calculus operators. J. Math. 2019, Article ID 9864737 (2019). https://doi.org/10.1155/2019/9864737

36. Haubold, H.J., Mathai, A.M., Saxena, R.K.: Mittag-Leffler functions and their applications. J. Appl. Math. 2011, Article ID $298628(2011)$

37. Gradshteyn, I.S., Ryzhik, I.M.: Tables of Integrals, Series, and Products. Academic Press, New York (1980)

\section{Submit your manuscript to a SpringerOpen ${ }^{\circ}$ journal and benefit from:}

- Convenient online submission

- Rigorous peer review

- Open access: articles freely available online

- High visibility within the field

- Retaining the copyright to your article

Submit your next manuscript at $\gg$ springeropen.com 